\title{
Experimental and Mathematical Analysis of Hepatic Uptake
}

\author{
T. GRANDJEAN ${ }^{1}$, A. LENCH ${ }^{2}$, J. W. T. YATES ${ }^{3}$, \\ M. J.CHAPPELL ${ }^{1}$, C. J. O'DONNELL ${ }^{3}$ \\ ${ }^{1}$ School of Engineering, University of Warwick, Coventry, CV4 7AL, United Kingdom \\ 2 Departments of Pharmacy and Pharmacology, University of Bath, Bath, United Kingdom \\ ${ }^{3}$ DMPK Department, Astrazeneca, Alderley Park, Macclesfield, United Kingdom \\ E-mail: james.yates@astrazeneca.com (J. Yates)
}

Sci Pharm. 2010; 78: 696

doi:10.3797/scipharm.cespt.8.PPAT07

\section{Introduction:}

To investigate the nonlinear kinetics of in vitro hepatic uptake the OATP substrate, Pitivastatin, was used as a probe. Experiments were conducted using freshly isolated rat hepatocytes, utilising the 'oil spin' methodology described by Hassen et al [1]. Briefly, freshly isolated rat hepatocytes were incubated with Pitvastatin $(5-300 \mu \mathrm{M})$. At $10 \mathrm{~s}, 30 \mathrm{~s}, 50 \mathrm{~s}$ and $70 \mathrm{~s}$ aliquots were spun through a silicone oil layer to separate the hepatocytes from the media. [Pitivastatin $]_{\text {hepatocyte }}$ was detemined using LCMSMS.

Results:

Uptake to rat hepatocytes was saturable and progressed according to Michaelis-Menten kinetics. The $\mathrm{K}_{\mathrm{m}}$ and $\mathrm{V}_{\max }$ of Pitvastatin were 2050 pmols $/ \mathrm{min} / 10^{6}$ cells and $33 \mu \mathrm{M}$ respectively, which was in in good agreement with other literature reports [2].

Mathematical Modelling:

A nonlinear pharmacokinetic model has been derived to characterise the uptake process. A structural identifiablity analysis was performed on the model to establish that all unknown parameters could be identified from the experimental observations available. The model was then subsequently used for parameter estimation and model validation using the data collected. Sensitivity analysis and model robustness analyses were also performed. Once fully validated the model has the potential to perform robust, predictive simulations to ascertain optimal levels of uptake and the effects of the use of appropriate inhibitors.

This work was supported by $A Z$ \& TG was supported by $A Z$ and the MRC Capacity Building initiative.

[1] Hassen AM, Lam D, Chiba M, Tan E, Geng W, Pang KS. Uptake of sulfate conjugates by isolated rat hepatocytes. Drug Metab Pharmacokin. 1996; 24: 792-798. PMid:8818578

[2] Shimada S, Fujino H, Morikawa T, Moriyasu M, Kojima J. Uptake mechanism of pitavastatin, a new inhibitor of HMG-CoA Reductase, in rat hepatocytes. Drug Metab Pharmacokin. 2003; 18: $245-251$. doi:10.2133/dmpk.18.245 